(c) Elsevier/INRA

\title{
Sélection divergente pour la consommation alimentaire résiduelle de la poule en période de ponte : réponse au taux protéique de l'aliment
}

\author{
A Bordas, P Mérat \\ Institut National de la Recherche Agronomique, laboratoire de génétique factorielle, \\ centre de recherches de Jouy-en-Josas \\ 78352 Jouy-en-Josas Cedex, France
}

(Reçu le 26 octobre 1990; accepté le 21 janvier 1991)

Résumé - Des poules de 2 lignées (respectivement $R^{+}$et $R^{-}$) sélectionnées pour augmenter ou diminuer la fraction résiduelle de la consommation alimentaire en période de ponte ont été réparties en 2 groupes (ingestion élevée/baisse de protéines brutes) recevant des rations de même teneur en énergie et en éléments essentiels non protéiques. La teneur des rations en protéines brutes était calculée en fonction de la consommation alimentaire dans les 2 lignées, de façon à assurer à ces lignées sensiblement le même niveau d'ingestion de protéines dans chaque groupe experimental.

Une ingestion journalière plus élevée de protéines entraîne un poids corporel, un poids moyen d'œufs et un pourcentage de coquille plus élevés, augmente le taux plasmatique d'acide urique, diminue la consommation alimentaire résiduelle et le poids du gésier en pourcentage du poids vif.

Les différences entre lignées $R^{+}$et $R^{-}$sont hautement significatives non seulement pour la consommation d'aliment ou sa fraction résiduelle et l'indice de consommation pour la ponte, mais aussi pour l'âge au $1^{\mathrm{er}}$ œuf, le pourcentage d'œufs mous, à 2 jaunes ou cassés, le poids du jaune, le poids corporel, le pourcentage du plumage et du gésier rapporté au poids vif, variables de moyenne plus élevée dans la lignée $R^{+}$, et pour le poids et le pourcentage de coquille dans l'œuf, supérieurs dans la lignée $R^{-}$. Ces différences ont déjà été observées antérieurement.

La consommation alimentaire des 2 lignées, y compris sa composante résiduelle, reste fortement différente quel que soit le niveau d'ingestion des protéines; cependant, l'apport protéique le plus faible s'accompagne d'un abaissement de la consommation alimentaire, du poids corporel et de l'intensité de ponte des poules $\left\langle R^{+}\right.$» et non des poules « $R^{-} 》$ (Interactions Lignée $\times$ Traitement significatives). Ces lignées diffèrent donc, indépendamment du niveau protéique, pour leur ingestion alimentaire et énergétique, mais il est possible d'autre part que la lignée $R^{+}$détourne plus de matières protéiques à des fins énergétiques que $R^{-}$.

poule / production d'œufs / sélection / efficacité alimentaire / taux protéique 
Summary - Divergent selection for residual feed consumption of the hen in the laying period : response to the protein level of the ration. Laying hens from 2 lines, selected for high and low residual component of feed intake in the laying period, $\left(\mathrm{R}^{+}\right.$and $\mathrm{R}^{-}$ respectively) were distributed in 2 groups (high/low ingestion of crude proteins) receiving rations of the same level in energy and non-proteinic essential components. The content of the rations in total protein was calculated according to the feed intake of the 2 lines so as to provide these lines with nearly the same daily amount of protein within each experimental group. A higher daily protein intake causes à higher body weight, mean egg weight, shell percentage and plasma uric acid level and a lower residual feed consumption and weight of gizzard as percent of body weight. The difference between the $\mathrm{R}^{+}$and $\mathrm{R}^{-}$lines is highly significant not only for feed intake, its residual component and feed efficiency of egg production, but also for age at first egg, percent of shell-less, double-yolked and cracked eggs, yolk weight, adult body weight, feathers and gizzard in percent of live weight, which are higher in the $\mathrm{R}^{+}$line, and for weight and percentage of shell in the egg, which are superior in $\mathrm{R}^{-}$. These differences are consistent with previous observations.

The feed intake of the 2 lines and its residual component remain considerably different irrespective of the level of protein intake; however, the lower protein supply is associated with a lower feed intake, body weight and laying rate for the $\mathrm{R}^{+}$but not for the $\mathrm{R}^{-}$layers (significant line $\times$ treatment interactions). Although these lines differ for their feed and energy intake at any protein level, it is likely that the $\mathrm{R}^{+}$line uses more protein than $\mathrm{R}^{-}$ for energy production.

domestic fowl / egg production / feed efficiency / protein level

\section{INTRODUCTION}

Une sélection divergente sur la consommation alimentaire «résiduelle» des oiseaux adultes a été poursuivie à partir d'une population de base Rhode Island depuis 1976. Les réponses directes et liées à cette sélection obtenues après 7 générations dans les lignées divergentes « $R^{+} 》$ (consommation élevée) et « $R^{-}$» (faible consommation) ont été présentées par Bordas et Mérat (1984), puis résumées en ce qui concerne la $10^{\mathrm{e}}$ génération par Tixier-Boichard et al (1988).

L'ingestion alimentaire différant considérablement entre ces 2 lignées, il en est de même des composants protéiques de la ration. En conséquence, nous avons voulu vérifier dans le présent travail les performances de ponte et l'efficacité alimentaire de poules de ces 2 lignées ramenées à un même niveau d'ingestion des protéines brutes.

\section{MATÉRIEL ET MÉTHODES}

\section{Animaux et conditions expérimentales}

Le 18 novembre 1988, une éclosion unique a donné 440 poussins de la lignée « $R^{+}$» et 430 poussins de la lignée « $R^{-}$». Les caractéristiques de ces lignées ont été décrites par ailleurs (Bordas et Mérat, 1984).

Les poussins étaient élevées au sol avec un aliment à $20 \%$ de protéines brutes et $2800 \mathrm{kcal} / \mathrm{kg}$ EM jusqu'à 10 semaines, puis $15,3 \%$ de protéines brutes et $2750 \mathrm{kcal} / \mathrm{kg}$ EM de 10 à 18 semaines. À l'âge de 18 semaines, un total de 
160 femelles (80 par lignée) étaient transférées en cages individuelles. À partir du même âge, les poules de chaque lignée étaient réparties en 2 groupes recevant chacun un aliment différent, tous 2 sous forme de farine.

Les caractéristiques principales et la composition des 3 aliments utilisés sont données au tableau I.

Tableau I. Composition des régimes.

\begin{tabular}{|c|c|c|c|}
\hline & \multicolumn{3}{|c|}{ Pourcentage par aliment } \\
\hline & Témoin & $X$ & $Y$ \\
\hline \multicolumn{4}{|l|}{ Composition } \\
\hline Maïs & 52,12 & 49,48 & 58,70 \\
\hline Son de blé & 5,58 & & 12,26 \\
\hline Pois & 7,87 & & 10,00 \\
\hline Tourteau soja $48 \%$ & 5,74 & 32,54 & 2,58 \\
\hline Tourteau tournesol & 7,00 & 1,63 & 1,13 \\
\hline Farine de viande dégraissée & 4,41 & 0,45 & 3,19 \\
\hline Luzerne & 0,76 & 3,03 & 5,65 \\
\hline Graisse animale & 0,49 & 1,00 & - \\
\hline Drèches de distillerie de maïs & 8,00 & - & - \\
\hline Phosphate bicalcique & - & 1,36 & - \\
\hline Carbonate de calcium & 7,03 & 9,48 & 5,55 \\
\hline Compl vitam et minér ${ }^{1}$ (V34 UFAC) & 1,00 & 1,00 & 0,50 \\
\hline Méthionine ${ }^{2}$ & & 0,04 & 0,13 \\
\hline Chlorure de sodium ${ }^{2}$ & & & 0,31 \\
\hline \multicolumn{4}{|l|}{ Caractéristiques nutritionnelles } \\
\hline $\mathrm{EM}(\mathrm{kcal} / \mathrm{kg})^{+}$ & 2674 & 2667 & 2692 \\
\hline $\operatorname{Eau}(\%)^{+}$ & 12,52 & 12,07 & 12,94 \\
\hline Matières protéiques brutes $(\%)^{*}$ & 16,41 & 20,87 & 13,48 \\
\hline Amidon $(\%)^{*}$ & 36,96 & 28,81 & 41,31 \\
\hline Cellulose $(\%)^{+}$ & 5,50 & 4,15 & 5,50 \\
\hline Matières minérales $(\%)^{*}$ & 12,73 & 14,23 & 10,20 \\
\hline Matières grasses $(\%)^{+}$ & 3,43 & 4,20 & 3,03 \\
\hline Phosphore disponible $(\%)^{+}$ & 0,34 & 0,43 & 0,27 \\
\hline Calcium* ${ }^{*}$ & 3,69 & $4 ; 34$ & 2,92 \\
\hline Méthionine $^{+}$ & 0,31 & 0,40 & 0,25 \\
\hline Lysine $^{+}$ & 0,67 & 0,85 & 0,54 \\
\hline Thréonine $^{+}$ & 0,56 & 0,79 & 0,47 \\
\hline Tryptophane $^{+}$ & 0,14 & 0,24 & 0,12 \\
\hline Acide linoléique $^{+}$ & 1,51 & 1,73 & 1,51 \\
\hline
\end{tabular}

${ }^{*}=$ résultat d'analyse ${ }^{+}=$calcul $;{ }^{1}=$ Incluant $\mathrm{ClNa}$ et méthionine $;^{2}=$ en supplément du complément précédent.

Ces rations étaient calculées de façon à avoir la même concentration en énergie métabolisable (EM). Leurs teneurs différentes en protéines brutes (ainsi qu'en acides aminés) étaient calculées en fonction de la différence de consommation alimentaire des poules des 2 lignées, observée à la génération précédente de façon que, d'une part 
les poules $R^{-}$recevant l'aliment $X$ (plus riche en protéines brutes) et les poules $R^{+}$ recevant l'aliment témoin, d'autre part les poules $R^{-}$recevant l'aliment témoin et les poules $R^{+}$recevant l'aliment $Y$ (moins riches en protéines) ingèrent journellement une quantité voisine de protéines. A posteriori, la mesure des consommations alimentaires dans chaque sous-groupe a vérifié cette prévision.

La température ambiante moyenne était de $22^{\circ} \mathrm{C}$ avec des fluctuations limitées à environ $2^{\circ} \mathrm{C}$, les poules recevaient $14 \mathrm{~h}$ d'éclairement artificiel par $24 \mathrm{~h}$.

\section{Mesures}

Les performances de ponte étaient contrôlées entre le $1^{\text {er }}$ œuf et l'âge de 45 semaines. Le nombre d'œufs, l'intensité de ponte (rapport du nombre d'œufs au nombre de j de contrôles depuis le $1^{\text {er }}$ œuf), la longueur moyenne des séries (jours successifs de ponte sans interruption) et le pourcentage de $\mathrm{j}$ de pauses (arrêts de ponte d'au moins $2 \mathrm{j}$ consécutifs) concernaient la période entière de contrôle, ainsi que les pourcentages d'œufs mous (sans coquille), à 2 jaunes ou cassés.

La consommation d'aliment et la masse d'œufs étaient mesurées sur une période de 28 j entre les âges de 28 et 32 semaines, ainsi que le poids corporel moyen et sa variation entre le début et la fin de la période. La consommation résiduelle en était déduite comme l'écart individuel à la consommation théorique obtenue à partir d'une équation de régression sur le poids corporel, sa variation et la masse d'œufs (Gous et al, 1978). L'exposant 0,5 était pris pour le poids corporel, des résultats antérieurs ayant montré sa validité (Bordas et Mérat, 1984). Les coefficients des équations obtenus dans les 2 lignées ne différant pas significativement, l'équation unique suivante a été utilisée, donnant (en g par $28 \mathrm{j}$ ) la consommation prédite $T$ en fonction du poids corporel moyen $(P)$, de sa variation $(\Delta P)$ et de la masse d'œufs $(E)$ :

$$
T=124,0 P^{0,5}+0,93 \Delta P+0,84 E-3328
$$

Le poids moyen de l'ocuf était mesuré sur la ponte de 2 semaines entre les âges de 30 et 32 semaines, le poids de l'albumen, du jaune, le poids et le pourcentage de coquille et le rapport jaune/albumen étaient mesurés sur 1 œuf par poule dans la même période. À la fin du contrôle, on constituait un échantillon de 20 poules par groupe qui étaient prélevées et abattues pour des mesures anatomiques (variables données en pourcentage du poids vif) : l'importance relative de la graisse abdominale et du gésier avait déjà été trouvée différente pour les lignées comparées (Zein-el-Dein et al, 1985); d'autre part, l'état d'engraissement peut différer en réponse au taux protéique de la ration (voir par exemple, Anonyme, 1984). Une prise de sang était faite en fin de contrôle sur toutes les poules pour évaluation du taux plasmatique de triglycérides (Kit Boehringer 70/912, test GPO. $\mathrm{PAP}$ ) et d'acide urique (test colorimétrique à l'uricase, Kit Boehringer 556718 565-24j). Les analyses concernant les aliments ont été faites par l'UFAC (95450, Vigny).

\section{Analyses statistiques}

L'analyse de variance faite sur ces données prend en considération les 2 facteurs à effet fixe «lignée» et «quantité de protéines totales ingérée», cette dernière étant 
voisine d'une part pour les groupes « $R^{+} »$, aliment témoin» et « $R^{-}$, aliment $X »$; d'autre part, pour les groupes « $R^{+}$, aliment $Y »$ et « $R^{-}$, aliment témoin. Les 4 sousgroupes peuvent donc se répartir en fonction des 2 facteurs de variation.

\section{RÉSULTATS}

Le tableau II présente les quantités ingérées des principaux éléments de la ration par j pour chacun des groupes expérimentaux, calculées à partir de la composition des aliments et de l'ingestion moyenne de chaque groupe.

Tableau II. Quantités journalières des principaux composants alimentaires ingérées pour chaque groupe

\begin{tabular}{lcccc}
\hline Composant & \multicolumn{4}{c}{ Lignée - Régime } \\
\cline { 2 - 5 } & $R^{-}, T$ & $R^{-}, X$ & $R^{+}, T$ & $R^{+}, Y$ \\
\hline Energie $(\mathrm{kcal} / \mathrm{j})$ & 282 & 278 & 349 & 330 \\
Protéines totales $(\mathrm{g} / \mathrm{j})$ & 17,3 & 21,7 & 21,4 & 16,5 \\
Méthionine $(\mathrm{g} / \mathrm{j})$ & 0,33 & 0,42 & 0,40 & 0,31 \\
Lysine $(\mathrm{g} / \mathrm{j})$ & 0,71 & 0,89 & 0,88 & 0,66 \\
Amidon $(\mathrm{g} / \mathrm{j})$ & 39,0 & 30,0 & 48,3 & 50,7 \\
Matières grasses $(\mathrm{g} / \mathrm{j})$ & 3,62 & 4,32 & 4,48 & 3,72 \\
Phosphore disponible (g/j) & 0,36 & 0,45 & 0,44 & 0,33 \\
Calcium $(\mathrm{g} / \mathrm{j})$ & 3,89 & 4,52 & 4,82 & 3,58 \\
\hline
\end{tabular}

Le tableau III indique les valeurs moyennes des autres variables par lignée et par régime. L'analyse de variance faite sur ces données prend en considération, comme indiqué ci-dessus, les 2 facteurs «lignée» et «niveau d'ingestion protéique» (élevé oul bas).

Les comparaisons entre moyennes prises 2 à 2 ne sont pas présentées in extenso. Les plus intéressantes paraissent être celles entre moyennes d'une même lignée avec les 2 niveaux d'ingestion protéique; nous indiquons ci-après les cas où ces moyennes sont significativement différentes pour chaque lignée :

- pour la lignée $R^{-}$, l'aliment $X$ (enrichi) ne differe significativement de l'aliment témoin que pour la fraction résiduelle de la consommation alimentaire $(P<0,05)$ et pour le taux d'acide urique plasmatique $(P<0,01)$;

- pour la lignée $R^{+}$, l'aliment $Y$ (pauvre) diffère significativement de l'aliment témoin pour la consommation d'aliment, le poids moyen de l'œuf, le taux d'acide urique plasmatique $(P<0,05)$ et le poids corporel $(P<0,001)$.

\section{DISCUSSION ET CONCLUSION}

\section{Effets de la quantité de protéines ingérées}

Un apport journalier plus grand de protéines entraîne pour l'une ou l'autre lignée un poids corporel et un poids moyen des œufs plus élevés; le taux plasmatique d'acide 


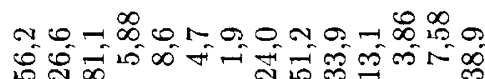

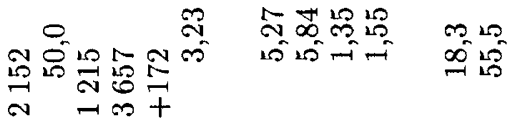

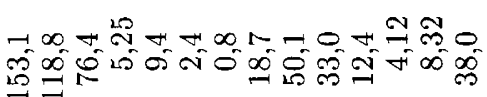

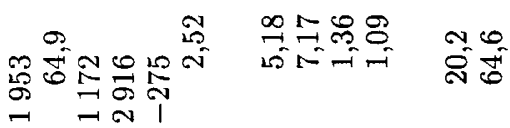
2

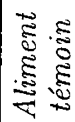

$\infty=\frac{10}{2}$ is 0 .

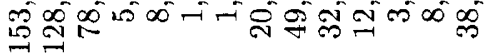

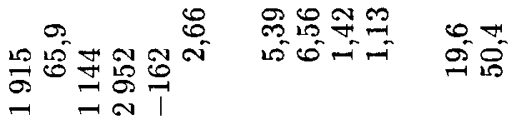
(2)

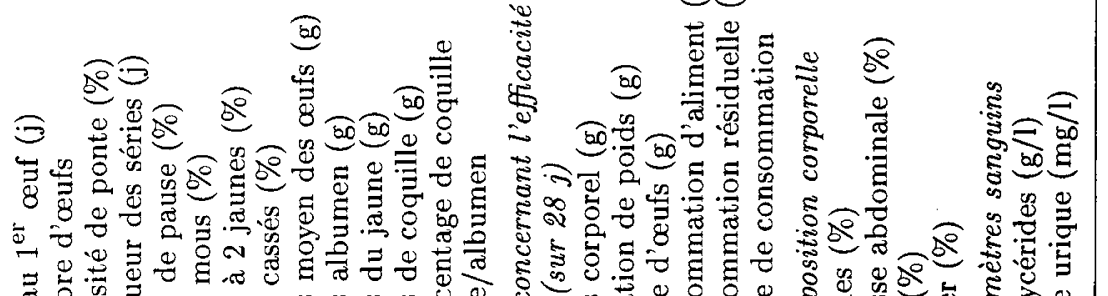
స్

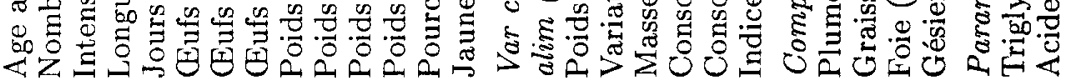


urique est augmenté comme on pouvait s'y attendre. Nous n'observons pas, sur notre échantillon limité, de différence significative de teneur en graisse abdominale, reflet supposé des lipides corporels. Un effet positif du taux protéique alimentaire sur le poids moyen des œufs a été observé fréquemment. Par contre la diminution de la consommation résiduelle d'aliment, celle du gésier en pourcentage du poids corporel et l'augmentation de la coquille de l'œuf en poids ou en pourcentage avec une ingestion protéique plus élevée ne semblent pas avoir été relatées auparavant et restent à interpréter.

\section{Différences entre lignées}

On retrouve entre les 2 lignées une différence importante sur le caractère pour lequel elles ont été sélectionnées dans un sens divergent (consommation résiduelle en période de ponte) et une différence similaire pour la consommation observée et l'indice de consommation pour la production d'œufs. On trouve d'autre part, dans l'ensemble, un poids corporel un peu supérieur pour les poules $R^{+}$. Pour les caractéristiques des œufs alors que le poids du jaune et celui de l'albumen sont plus élevés pour les poules $R^{+}$, le poids et le pourcentage de coquille sont significativement supérieurs chez les poules $R^{-}$. D'autre part, les poules $R^{-}$, plus faibles consommatrices, ont moins d'œufs mous, à 2 jaunes ou cassés, sont de quelques jours plus précoces pour l'entrée en ponte, ont un poids de plumage légèrement inférieur au total et le poids de leur gésier rapporté au poids corporel est considérablement inférieur, peut être en relation avec la différence d'ingestion alimentaire. Les différences observées dans la présente comparaison concordent en tous points avec celles notées dans les générations de sélection successives (Bordas et Mérat, 1984; Zein-el-Dein et al, 1985; Tixier-Boichard et al, 1988; Bordas et Mérat, données non publiées; en ce qui concerne la composition corporelle). D'un point de vue pratique, la meilleure qualité des coquilles et la réduction des œufs cassés ou mous peuvent renforcer l'intérêt potentiel de la lignée plus «économe» d'aliments.

\section{Réponses comparées des lignées au niveau d'ingestion des protéines}

La consommation alimentaire dans les 2 lignées, y compris sa fraction résiduelle, reste fortement différente quel que soit le niveau d'ingestion des protéines brutes. Le changement du niveau d'ingestion de protéines ne modifie pas la différence de consommation résiduelle entre les 2 lignées. On observe cependant une interaction lignée $\times$ régime pour la consommation observée; la différence entre lignées est moindre avec l'apport protéique le plus faible, qui s'accompagne d'un abaissement de la consommation des poules $R^{+}$et non des poules $R^{-}$. En relation avec cette réduction de consommation des poules $R^{+}$en présence d'un aliment moins riche en protéines, leur poids corporel est diminué par rapport à l'aliment témoin alors que les poules $R^{-}$ne sont pratiquement pas affectées par le niveau protéique. La ponte paraît également diminuée pour les poules $R^{+}$recevant moins de protéines totales, et par contre n'est pas augmentée, mais plutôt abaissée, pour les poules $R^{-}$consommant plus de protéines, par rapport à celles recevant l'aliment témoin. Ces dernières ne sont donc pas limitées dans leurs performances par la quantité 
de protéines dont elles disposent; une quantité de $17,3 \mathrm{~g} / \mathrm{j}$ est suffisante pour leur assurer une production maximale.

L'augmentation du taux plasmatique d'acide urique avec la quantité de protéines ingérées, aussi bien pour les pondeuses $R^{-}$que pour les $R^{+}$, suggère d'ailleurs qu'un apport protéique supplémentaire n'est pas utilisé davantage par les poules faibles consommatrices que par les autres. Par contre, le passage à un aliment plus riche en protéines brutes se traduit par une augmentation de l'intensité de ponte et du poids corporel chez les poules $R^{+}$fortes consommatrices d'énergie : elles doivent consommer plus de $20 \mathrm{~g} / \mathrm{j}$ de protéines brutes pour donner une ponte maximale. En conclusion, si l'augmentation du taux protéicue alimentaire n'avantage pas les poules $R^{-}$sous-consommatrices, inversement un faible taux protéique n'est pas mieux supporté par les poules fortes consommatrices que par les autres. La différence entre lignées concerne essentiellement l'ingestion alimentaire, mais si l'on suppose une appétence et une digestibilité égales des différentes rations, on peut supposer que la lignée $R^{+}$détourne plus de protéines à des fins énergétiques que $R^{-}$et que cet aspect métabolique serait prioritaire sur la production d'œufs. Divers travaux chez le poulet de chair, passés en revue par Pym (1990), indiquent une utilisation plus importante des protéines à des fins énergétiques dans des lignées moins efficientes pour leur conversion alimentaire, par exemple des lignées grasses, que dans les autres. D'un point de vue pratique, une modification de la ration, du moins quant à son niveau protéique, laisse essenticllement inchangé l'avantage économique global de la lignée faible consommatrice.

\section{REMERCIEMENTS}

Nous remercions M Picard (INRA, station de recherches avicoles, 37380 Monnaie) pour ses suggestions et conseils dans l'établissement des rations et l'interprétation des résultats.

\section{RÉFÉRENCES}

Anonyme (1984) L'alimentation des animaux monogastriques : porcs, lapins, volailles (Blum JC, ed) INRA, Paris

Bordas A, Mérat P (1984) Réponses liées dans une expérience de sélection sur la consommation alimentaire «résiduelle» de coqs et poules Rhode Island. 17 Congr Mondial d'Aviculture, Helsinki, 106-108

Gous RM, Byerly TC, Thomas OP, Kessler JM (1978) A partition equation to predict food and energy intake by laying hens. $10^{\circ}$ Congr Mondial d'Aviculture, Rio de Janciro, II, 1-8

Pym RAE (1990) Nutritional genetics. In : Poultry Breeding and Genetics (Crawford RD, ed), Elsevier, Amsterdam, 847-876

Tixier-Boichard M, Bordas A, Mérat P (1988) Divergent selection for residual feed intake in laying hens : effects on growth and fatness. In : Leanness in Domestic Birds (Leclercq, Whitehead CC, eds), INRA - Butterworths, London, 129-132 Zcin-El-Dein A, Bordas A, Mérat P (1985) Sélection divergente pour la composante «résiduclle» de la consommation alimentaire des poules pondeuses : effets sur la composition corporclle. Arch Gefluegelde 49, 158-160 